\title{
Mutations of Drosophila melanogaster that affect muscles
}

\author{
By I. I. DEAK ${ }^{1}$ \\ From the Division of Biology, California Institute of Technology, \\ Pasadena, California, U.S.A.
}

\begin{abstract}
SUMMAR Y
Eight $X$-chromosome mutations (falling into five complementation groups) that affect the development and morphology of the indirect flight muscles of Drosophila melanogaster were investigated using histological, behavioural and genetic techniques. All of these mutations result in flightlessness, in a marked reduction in the ability of the flies to jump, and in the wings being held in abnormal positions. Mutations in each of the complementation groups have different effects on the morphology of the muscles. Two (flapwing, vertical wing) result in absence of most of the indirect flight muscle fibres, a third (upheld) is required for the gross organization of muscle structure, another (heldup) is involved in the maintenance of muscle structure once formed, and the fifth seems to be necessary for the detailed architecture of the muscle fibre (indented thorax). The analysis of flies genetically mosaic with respect to each mutation by the technique of fate-mapping suggests that three (heldup, upheld and indented thorax) of the genes concerned have their primary site of action in the musculature itself, while the other two (flapwing and vertical wing) may function primarily in the fat-body and tracheae respectively.
\end{abstract}

\section{INTRODUCTION}

The anatomy and the morphology of the musculature of Drosophila melanogaster have been described in detail (Behrendt, 1940; Tiegs, 1955; Shafiq, 1963; Miller, 1965). The muscles can be subdivided into fibrillar and tubular groups on morphological grounds (Snodgrass, 1935). The indirect flight muscles, which are fibrillar, constitute the bulk of the muscle mass in the thorax and provide the propulsive force during flight. They also control the shape of the thoracic cavity and hence the position of the wings (Pringle, 1957). Each fibre is a syncytium containing 2000-2400 nuclei (Tiegs, 1955) and results from fusion of myoblasts during pupal development (Tiegs, 1955). It contains a highly ordered array of myofibrils that run the length of the fibres. The fibres fall into two groups that can be differentiated on the basis of their position and orientation in the thorax. These are the dorsal longitudinal fibres (DLMs) and the dorsoventral fibres (DVMs). There are 26 fibrillar fibres in each fly.

The rest of the muscles are of tubular type. They are generally small and

${ }^{1}$ Present address: Zoologisches Institut der Universität Zürich, Künstlergasse 16, 8006 Zürich, Switzerland. 
consist of a number of fibres arranged cylindrically. The largest tubular muscle is the tergal depressor of the trochanter of the second leg (TDT), which is responsible for jumping (Behrendt, 1940).

The fibrillar muscles differ from the tubular muscles not only on morphological grounds but also histochemically (Deak, 1976a). In particular, the fibrillar muscles contain large quantities of glycogen, while the TDT is devoid of glycogen (Deak, 1976b).

Little is known about the development of the indirect flight muscles of Drosophila. One way in which to investigate the development of these muscles would be to obtain mutations that result in abnormal muscle development. Study of the effects of such mutations could provide information as to the role of normal gene expression in muscle development.

When examining mutations affecting muscle it is essential to know whether they have their primary effect on the muscles indirectly by causing the environment in which the muscles develop to be defective. For example, some mutations might change the composition of the haemolymph by eliminating factors essential for muscle development, while others may prevent the muscles from developing normally by causing abnormal innervation or tracheation. The study of flies genetically mosaic with respect to any mutation allows one to distinguish between a primary and a secondary effect on muscle development, and to establish in which tissue the mutation has its primary effect.

This paper describes a number of $X$-chromosome mutations of Drosophila melanogaster that affect the indirect flight muscles. These mutations have been examined using genetic, histological, histochemical and behavioural techniques. They have also been studied in mosaic flies, and subjected to fate-mapping analysis (Sturtevant, 1929; Garcia-Bellido \& Merriam, 1969) to identify the primary site of action of the defective genes (Hotta \& Benzer, 1972).

\section{MATERIALS AND METHODS}

\section{Flies}

The Canton-S (C-S) strain of flies was taken as normal. Mutant stocks were kept balanced over the first chromosome balancer $\operatorname{In}(1) F M 7 b, y^{31 d} W^{a} l z^{s} B$ (Merriam, 1969; see Lindsley and Grell, 1968, for a description of mutations and chromosomes).

All stocks of flies were maintained at $25^{\circ} \mathrm{C}$. All of the mutant lines were also kept at $29^{\circ} \mathrm{C}$ and at $18{ }^{\circ} \mathrm{C}$ throughout development, to see whether in any of them expression of the mutant characteristic was dependent on temperature.

All flies were examined 5-8 days after emergence; at this time the muscles of C-S flies attain a maximum level of various enzymes and of glycogen (Deak, $1976 a$ ). For the examination of pupae animals were collected as white prepupae and kept at $25^{\circ} \mathrm{C}$. 


\section{Mutations}

The muscle mutants were obtained from several different sources; these will be referred to in the description of each mutant. Those mutants isolated at the California Institute of Technology were induced by ethylmethanesulphonate (EMS) as described by Lewis \& Bacher (1968). Mutations were mapped using the multiply-marked $X$-chromosomes y cho cv sn $f, y$ cho $v f y^{+}$, or $y$ cho $H k f$ car (Hk is the behavioural mutant, Hyperkinetic (Kaplan \& Trout, 1969)).

\section{Viability}

C-S and mutant males were crossed to virgin $C(I) R M, y f$ females and the ratio of male to female offspring for each mutant compared with the equivalent ratio obtained for C-S flies, which gave a male to female ratio of 1.56:1 (873 flies). All viability estimates presented are based on approximately 500 progeny.

\section{Morphological and histochemical examination}

\section{(a) Frozen sections for histochemistry}

Flies or pupae were sectioned using a SLEE model HR cryotome (SLEE International, Inc., New York, N.Y.). They were placed in a droplet of TissueTek O.C.T. compound (Ames Co., Elkhardt, Ind.) on a SLEE specimen holder, and rapidly frozen in a SLEE $\mathrm{CO}_{2}$ bench freezer. The resulting block was trimmed to a rectangular shape approximately $3 \mathrm{~mm} \times 4 \mathrm{~mm}$. The specimen holder was then placed in the cryotome and $15 \mu \mathrm{m}$ sections cut. Ribbons of serial sections were picked up on glass microscope slides and allowed to air-dry.

Sections were fixed in $10 \%$ formalin for $5 \mathrm{~min}$ at room temperature, and then stained for glycogen using a silver-staining technique (Deak, 1976b) modified from a stain for degenerating nerve terminals developed by Roth, Gomez, Voneida \& Edwards (1971).

Other sections were stained for various enzymes (malate, succinate, isocitrate and $\alpha$-glycerophosphate dehydrogenases, and myosin adenosine triphosphatase) as described by Deak (1976a).

\section{(b) Histology}

Fly thoraces were fixed in Bouin's fluid, and then silver-stained according to the Bielchowsky technique. Fixed thoraces were rinsed in $0.6 \mathrm{M}-\mathrm{NaNO}_{3}$, left in an ammoniacal ethanol solution $\left(2 \mathrm{ml} \mathrm{NH}_{4} \mathrm{OH}\right.$ in $\left.100 \mathrm{ml} 95 \% \mathrm{C}_{2} \mathrm{H}_{5} \mathrm{OH}\right)$ for $3 \mathrm{~h}$, rinsed again in $0.6 \mathrm{M}-\mathrm{NaNO}_{3}$, and left for $24 \mathrm{~h}$ in $10 \% \mathrm{AgNO}_{3}$. They were then placed for $9 \mathrm{~h}$ in ammoniacal silver $\left(7 \mathrm{ml} 0.5 \mathrm{M}-\mathrm{NaNO}_{3}, 30 \mu 140 \%\right.$ $\mathrm{NaOH}, 130 \mu 1 \mathrm{NH}_{4} \mathrm{OH}, 1 \mathrm{ml} 10 \% \mathrm{AgNO}_{3}$; the latter added dropwise until the solution is unstable, i.e. until a drop of the solution when placed on filterpaper turns black within $1 \mathrm{~min}$ ), followed by exposure for $1 \mathrm{~h}$ to a developing solution (10 $\mathrm{ml} \mathrm{9 \%} \mathrm{Na}_{2} \mathrm{~S}_{2} \mathrm{O}_{3}, 1 \mathrm{ml} 5.5 \% \mathrm{AgNO}_{3}$ and $0.5 \mathrm{ml} 0.5 \%$ hydroquinone). This was followed by washing the pieces for $10 \mathrm{~min}$ in distilled water, and 
then by passing them through increasing concentrations of ethanol and finally transferring to xylene. The dehydrated thoraces were then embedded in Epon (Epon 812:dodecylsuccinicanhydride:nadicmethyl anhydride:DMP-30 in the proportions 16:11:10:1; these reagents were obtained from Polysciences, Rydsall, Penn.) for $8 \mathrm{~h}$ at $60^{\circ} \mathrm{C}$. The blocks obtained were sectioned with a Sorvall Porter-Blum MT2-B ultramicrotome (Sorvall, Newtown, Conn.) at $2 \mu \mathrm{m}$.

\section{(a) Flight}

\section{Behaviour testing}

Flies were tested for flight by a modification of Benzer's (1973) method. Flies were dropped individually into a $1000 \mathrm{ml}$ measuring cylinder. Since the flies were to be recovered after testing, the sides of the cylinder were not coated with oil. Each fly was tested five times. Flies falling to the bottom of the cylinder were scored as flightless, flies that reached the wall of the cylinder near the top were recorded as being able to fly, and those that consistently reached the cylinder wall half-way down were considered to have intermediate flight ability: when allowed to fly in an open space they did not fly more than $25 \mathrm{~cm}$.

\section{(b) Jump}

After testing the flies for flight the wings were removed and the flies tested for their ability to jump in response to a light touch on the abdomen. Flies were classified as non-jumping, jumping weakly (less than $2 \mathrm{~cm}$ ) or jumping normally (a distance of 3-7 cm, like C-S).

\section{Production and handling of mosaic flies}

Production of mosaic flies involved the use of the unstable ring- $X$ chromosome, $\operatorname{In}(1) w^{v C}$. Virgin females heterozygous for this chromosome were crossed to males in which any of the mutations affecting muscle $(m)$ were linked to the markers $y$ and cho or $w$. Loss of the ring- $X$ chromosome from the ensuing $\operatorname{In}(1) w^{v C} / y$ cho $m$ female zygotes results in the appearance of mosaic flies, in which the male cuticle is marked with $y$ and cho or $w$, while the female cuticle is phenotypically normal.

The mosaic progeny were collected, and each fly etherized and the distribution of male and female cuticle recorded. One day later its external morphology (i.e. wing positions, leg or thoracic abnormalities, cuticle defects) and its behaviour (ability to fly, jump, etc.) were scored. $\operatorname{In}(l) w^{r C} / y$ cho mosaic flies similarly treated behaved like wild-type (C-S) flies in all respects. Five days later each fly was sectioned and stained for glycogen. The stained muscle fibres were examined and recorded as normal or abnormal, the criteria for abnormality depending on the mutant being investigated (see below).

\section{Fate-map analysis}

The data from mosaic flies for cuticular genotype, external appearance, muscle morphology and behaviour were collected for each mutation. Values for 
the frequencies with which selected structures (e.g. cuticular landmarks, muscle fibres) of different genotype were obtained, and were represented as distances on a fate-map. Since behaviour, such as the ability to fly, is a single parameter, but results from the interaction between at least two bilaterally symmetrical foci, it had to be analysed according to models for interacting foci. Two such models, i.e. that the behavioural foci of the mutations were 'domineering' or 'submissive' (Hotta \& Benzer, 1972), were used for these calculations.

The data obtained for the various mutants were treated according to the formulae of Hotta \& Benzer to obtain the distances on the fate-map of the behavioural foci from a number of cuticular sites and from the muscle abnormality foci. 'Domineering' foci can be distinguished from 'submissive' foci in that when the behavioural defect is 'domineering' a majority of the mosaic individuals show a mutant phenotype, and most of the flies whose cuticle is bilaterally mosaic have mutant behaviour, while when 'submissive' the mutant behaviour is only expressed in a minority of flies. Furthermore, the distances of the behavioural foci to the midline are found to be negative when the 'domineering' model is applied to a 'submissive' focus, and vice versa. The use of the 'domineering' and 'submissive' models depends on the mosaics being, on the average, half-male and half-female. This was true for the mosaics of all five mutants tested.

\section{RESULTS}

For comparison with the sections of mutant flies presented below, the indirect flight muscles and the TDTs of wild-type flies are shown in Figs. 1-3. The dorsal longitudinal fibres (DLMs) are numbered 1-6 (Fig. 1A), the dorsoventral fibres (DVMs) numbered 7-13 (Fig. 1B). Figs. 2 and 3 show longitudinal sections through the thorax of wild-type flies, stained with the Bielchowsky silver stain (Fig. 2), the Periodic-acid-Schiff's stain for glycogen (Fig. 3A), and the Deak $(1976 b)$ silver-staining technique for glycogen used in this paper (Fig. 3B).

(A) heldup (hdp)

\section{Characterization of muscle mutants}

Hotta \& Benzer (1972) isolated and described a mutation which they referred to as wingsup $A$. This mutation maps to $1-59$ and is therefore probably allelic to the mutation heldup ( $h d p$ ) mapping at this position and with the same phenotype (Lindsley \& Grell, 1968). The mutation $h d p$ has been lost and so cannot be tested for allelism or compared to wingsup $A$; however, wingsup $A$ will henceforth be referred to here as heldup $p^{2} . h d p^{2}$ is a recessive mutation, with $100 \%$ penetrance, and $65 \%$ viability.

Phenotype of heldup ${ }^{2}$

$h d p^{2}$ flies hold their wings up vertically but appear otherwise normal, except that in about $5 \%$ of the flies one or more of the femurs are sharply bent. The 


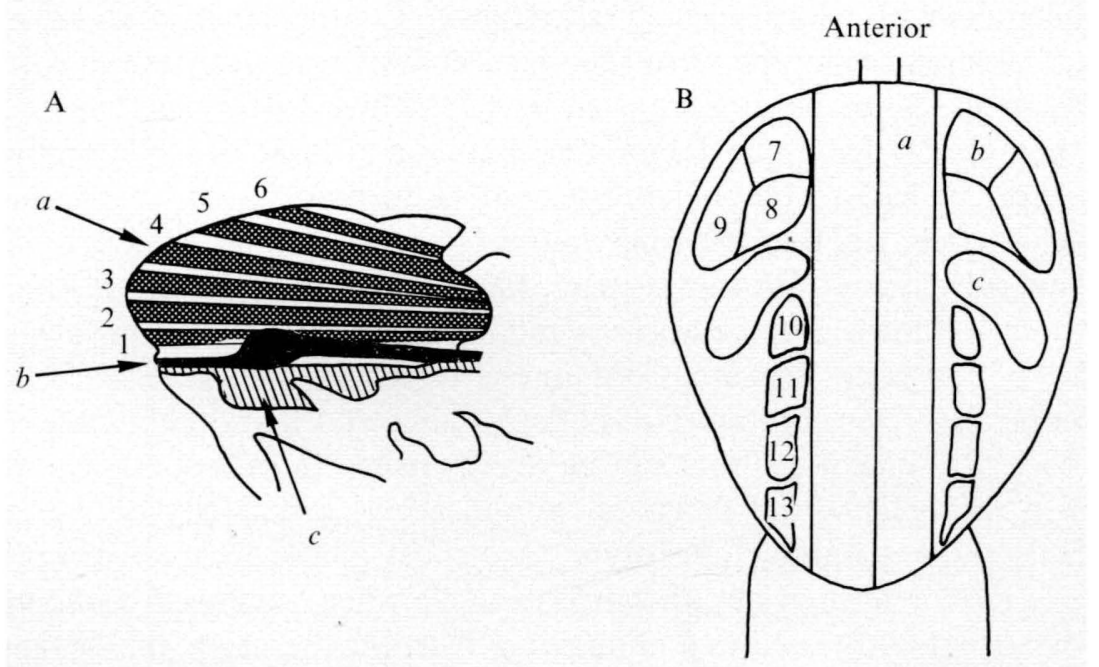

Fig. 1. (A) Diagrammatic sagittal section through the thorax of a normal fly. $a$, dorsal longitudinal indirect flight muscle fibres, numbered $1-6 ; b$, oesophagus, cardia and thoracic ventriculus; $c$, ventral nervous system. $\times 67$. (B) Diagrammatic longitudinal section through the thorax of a normal fly. $a$, dorsal longitudinal indirect flight muscle fibres; $b$, dorsoventral indirect flight muscle fibres, numbered $7-13 ; c$, tergal depressor of the trochanter (TDT). $\times 100$.

mutant is flightless, does not jump, and walks weakly. On excitation (banging and shaking the vial in which the fly is kept) the legs become very weak and the mesothoracic legs are folded under the body; the flies walk on their pro- and metathoracic legs. When heldup ${ }^{2}$ flies are etherized the mesothoracic legs are extended, unlike normal flies in which all three pairs of legs are folded up at the sides of the thorax.

The indirect flight muscles are largely absent (Hotta \& Benzer, 1972; Fig. 4).

Fig. 2. Longitudinal section through the thorax of a wild-type fly. Specimen Bielchowsky silver-stained and sectioned at $2 \mu \mathrm{m} . \times 90$.

Fig. 3. (A) Longitudinal section through the thorax of a wild-type fly. Frozen section stained with the Periodic-acid-Schiff's technique. The indirect flight muscles stain strongly for glycogen whereas no glycogen is found in the TDTs. $\times 110$. (B) Longitudinal section through the thorax of a wild-type fly. Frozen section stained with Deak's (1976b) silver-staining technique for glycogen.

Fig. 4. Longitudinal section through the thorax of a heldup ${ }^{2}$ fly. Fly frozen-sectioned and silver-stained for the presence of glycogen. The dorsal longitudinal indirect flight muscles are lacking, except for their posterior insertions. The anterior dorsoventral fibres are also absent. Some of the posterior dorsoventral fibres are present, but, with the exception of one heavily stained fibre (small arrow), lack glycogen. The TDTs are very swollen (large arrow). $\times 85$.

Fig. 5. Longitudinal section through the thorax of a bilatcrally mosaic $y$ cho $h d p^{2} j$ $\operatorname{In}(1) w^{\mathrm{vC}}$ gynandromorph. Specimen frozen-sectioned and silver-stained. The musculature of the left side appears totally normal, while that of the right side appears grossly abnormal. $\times 80$. 


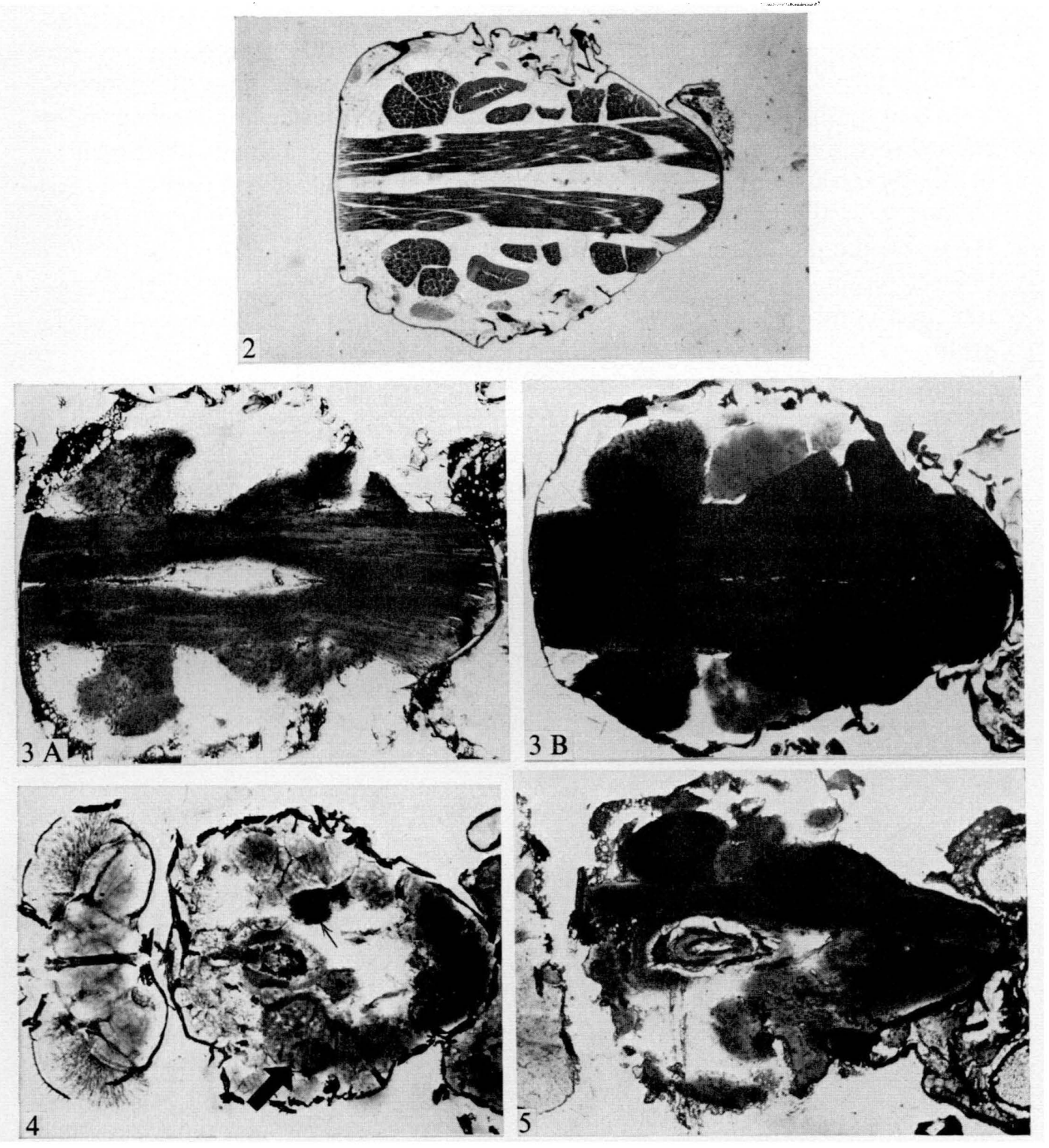

Only the insertions of the dorsal longitudinal fibres were present, much more material being found posteriorly. Occasionally fibres 5 and 6 were present, although very abnormal. In all flies DVMs 7, 8 and 9 were absent, while some of the posterior DVMs (10-13) were found in many of the flies.

Apart from the occasional fibre (approximately one fibre per two flies (Fig. 4)), all the fibres that remained in mutant flies lacked glycogen and diffusible enzymes such as malate and $\alpha$-glycerophosphate dehydrogenases (as visualized histochemically). 
The absence of soluble components such as glycogen may be due to the leakage of these components from the muscle; insoluble enzymes such as myosin adenosine triphosphatase were present in normal levels in the muscle fragments, as judged by the intensity of staining per unit area of muscle in histochemically stained sections. The lack of glycogen in the fibres was not due to the inability of the fly as a whole to synthesize glycogen; the level of glycogen in other structures in the body (halteres, abdomen, midgut, and cardia) was normal. However, the mutant may be unable to synthesize glycogen in its muscles.

Electron-microscopic examination shows that the muscle fragments that remain contain sarcomeres, with thick and thin filaments and Z-bands, although all these structures are grossly distorted (Hotta \& Benzer, 1972, 1973).

$h d p^{2}$ causes degeneration of the flight muscles late in pupal development (Hotta \& Benzer, 1972, 1973). During the first $60 \mathrm{~h}$ after pupariation the muscles appear to develop normally; at $60 \mathrm{~h}$ the indirect flight muscle fibres extend to approximately three-quarters of the length of the thorax. After this time they degenerate rapidly so that by emergence little of the flight musculature remains. No degeneration could be detected in adult flies.

The TDTs are usually very abnormal: the fibres are very swollen and their structure disorganized. The fibres are detached from each other, are rounded in cross-section, and the normally hollow core is filled with material. In some flies the TDTs are absent. In a few of the flies (1-2\%) they are indistinguishable from those of normal flies at the light-microscope level.

\section{Mosaics of heldup}

\section{(a) Fate-mapping of muscles: scoring criteria}

Using $y$ and cho as markers, 364 flies mosaic for $h d p^{2}$ were produced, and were handled as described in the Methods section. Figure 5 shows a section through the thorax of one such mosaic fly. Each of the fibres of each fly was examined and scored as normal or defective by its presence or absence, its normal or abnormal appearance, and its glycogen content. In C-S flies approximately $0.1 \%$ of the indirect flight muscle fibres (nine fibres in 352 flies, i.e. 9152 fibres examined) lacked glycogen. This level was so low that it was ignored in calculation of the fate-map distances. Individual fibres may themselves be mosaic: some fibres were reduced in diameter or in length, or had intermediate levels of glycogen. Very few such fibres were found in mutant flies. The TDT was scored as normal or abnormal according to its morphology.

The data obtained from examination of the muscles were compiled and analysed and fate-maps for the muscle abnormalities drawn (Figs. 6, 7). The indirect flight muscle fibres, and the TDTs, were taken as points for this analysis. Since each fibre is a syncytium, probably of non-clonal origin, taking the fibres as points represents an approximation which may introduce a certain amount of error. 
There are two factors that introduce errors into calculation of the fate-map. Because of these the details of the fate-maps, and the precise distances given, cannot be taken as definitive.

The first difficulty is that of establishing the criteria for scoring a fibre as normal or abnormal. Four separate fate-maps were drawn using different criteria. These included the two extreme possibilities: (1) the fibres were scored as abnormal unless they were totally normal, or (2) fibres were scored as normal unless they were absent. In the former case $63 \%$ of the fibres were scored as abnormal, in the latter $34 \%$ were thus scored. It is clear that neither of these criteria are really suitable, since fibres that appear intact but have slightly reduced levels of glycogen cannot be scored as totally abnormal (as they would in the first case), nor can fibres that are evidently grossly abnormal and lack glycogen be scored as normal (as they would in the second case).

The third possibility, therefore, was to make criteria that were a composite of the two criteria above, with the additional statement that each fibre $(29 \%$ of the total) that was neither totally normal nor absent was given an intermediate value ranging from one to four depending on the extent of its abnormality.

The fourth calculation was based on a similar approach, with the criteria somewhat altered. All fibres that were completely normal, or largely so, i.e. contained almost normal levels of glycogen and were not markedly reduced in size, were scored as normal; all fibres that were absent, or very much reduced in size, and had at most low levels of glycogen, were taken as abnormal; and the fibres ( $8 \%$ of the total) that could not categorically be placed in either category were put into a single intermediate class. Such intermediate cases were given a value of a half difference with respect to each cuticular landmark, whether male or female.

The fate-maps obtained using any of these four criteria were similar with respect to the position of the muscle abnormality focus relative to the cuticular structures and to the ventral midline, but there were considerable differences in the positions of the indirect flight muscle fibre foci relative to each other. Only small differences in relative positions and in sturt distances were obtained when the third or the fourth criteria were applied. The fate-maps presented here (Figs. $6,7)$ were calculated using the fourth set of criteria.

The second difficulty was that of incomplete penetrance of the mutations at the level of individual fibres. In $h d p^{2}$ flies an occasional fibre contains glycogen and appears normal in the light microscope; such fibres although genetically mutant would be scored as normal. This difficulty only arises when the posterior DVMs are mapped, since it is only in these that fibres sometimes appear normal. A similar problem arises when the TDTs are scored: $1-2 \%$ of the TDTs of heldup flies were indistinguishable from those of normal flies.

This problem can be corrected for in one of two ways. Instead of calculating (as is done in the case of complete penetrance) the proportion of flies in which fibre $a$ is scored as different from fibre $b$, regardless of which is normal and which 


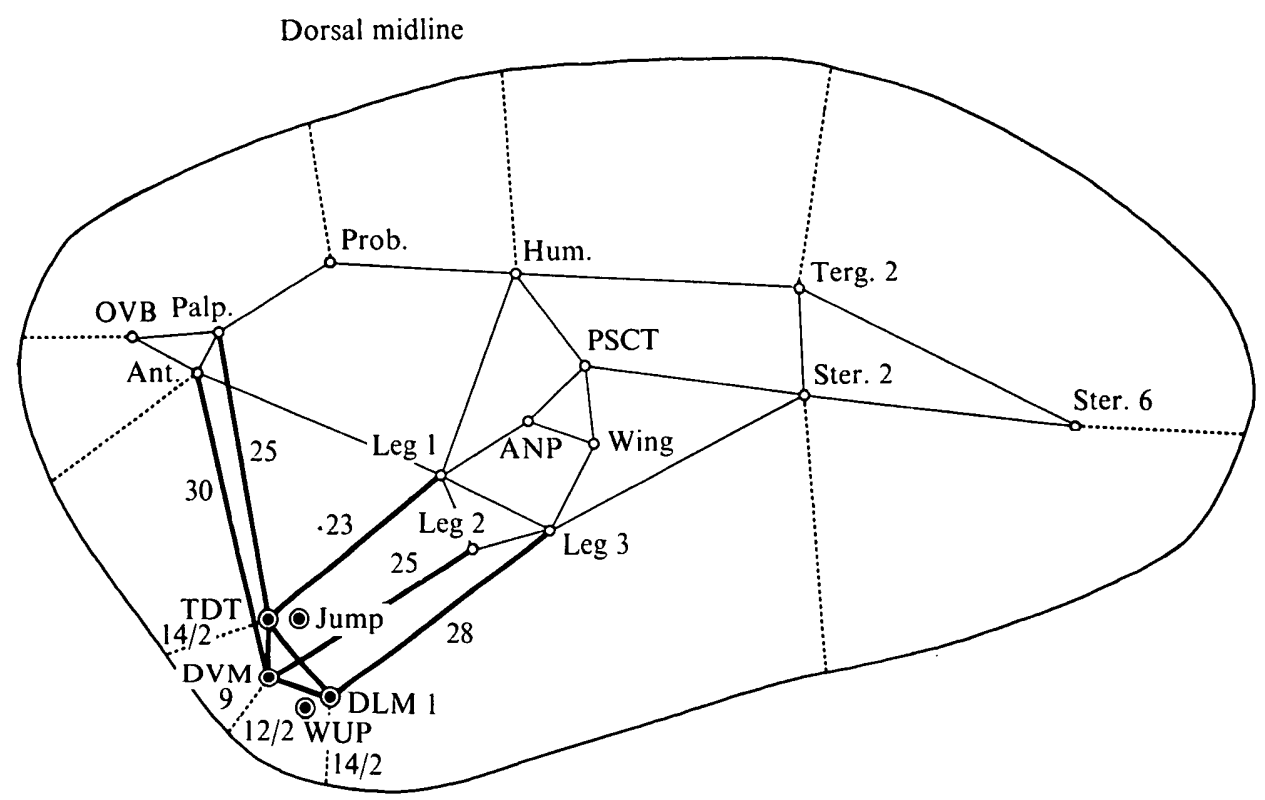

Ventral midline

Fig. 6. Fate-map of the behavioural and anatomical defects of the mutant heldup ${ }^{2}$. $\bigcirc$, cuticular landmarks; $\odot$, foci for abnormal behaviours and morphology. Values given are the distances in sturts from the foci to various cuticular landmarks and to the ventral midline. OVB, outer vertical bristle; Palp, palpus; Ant, third antennal segment; Prob; proboscis; Hum. humerus; L.eg 1, coxa cf first leg; Leg 2, coxa of second leg; Leg 3, coxa of third leg; PSCT, post cutellar bristle; ANP, anterior notopleural bristle; Wing, anterior edge wing blade; Terg 2, second abdominal tergite; Ster 2. second abdominal sternite; Ster 6, sixth abdomınal sternite; TDT: tergal depressor of the trochanter of the second leg; DVM 9, dorsoventral fibre no. 9; DML 1, dorsolongitudinal fibre no. 1; WUP, upheld wing position; Jump, inability to jump.

abnormal, only those cases in which fibre $a$ is abnormal and $b$ is normal are counted. This eliminates those situations in which fibre $a$ is incorrectly scored as normal when it is actually mutant. Such unidirectional scoring gives the same results as normal scoring when penetrance is complete.

Alternatively one can obtain a value for the extent to which penetrance is incomplete for each fibre, i.e. the proportion of fibres that are incorrectly scored in mutant flies, and subtract that value from the actual value obtained from mosaic flies. In this alternative the value for the degree of incomplete penetrance used depends on the criteria taken to score a fibre as normal or abnormal.

Neither of these corrections was entirely satisfactory but since they gave similar results either could be used. As the latter is more subject to variations in sampling the former was applied in all cases. 


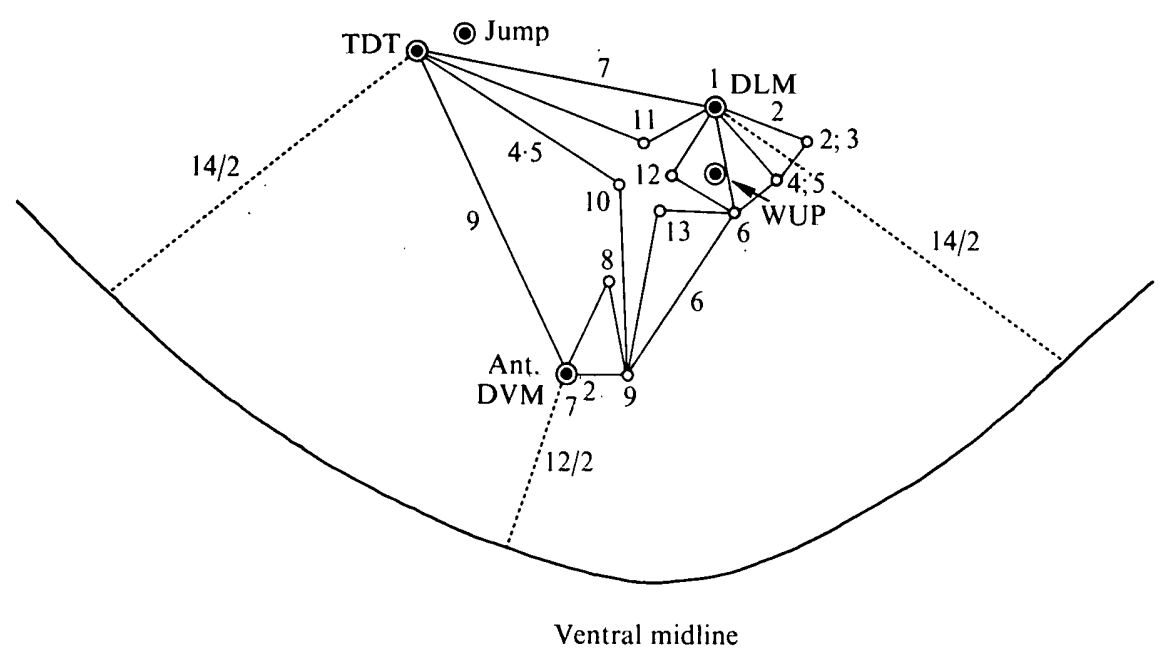

Fig. 7. Detailed fate-map of the behavioural and anatomical defects of the mutant heldup ${ }^{2}$. Small numerals correspond to the numbering of each indirect flight muscle fibre, large numerals correspond to the distances in sturts between each point. The behaviours, one dorsal longitudinal and one dorsoventral fibre, and the TDT have been emphasized $(\odot)$ for the sake of clarity. Lettering as in Fig. 6. Ant. DVM, anterior dorso-ventral fibres (7-9).

\section{(b) Fate-mapping of muscles of heldup ${ }^{2}$ : results and discussion}

The fate-maps presented in Figs. 6 and 7 show that the foci obtained for all of the mutant characteristics of heldup ${ }^{2}$ lie in the same part of the fate-map, anteroventral to the thoracic cuticle and close to the ventral midline. This agrees with the fate-map results obtained by Hotta \& Benzer (1972) for the behaviour of this mutant. This region of the fate-map probably corresponds to the ventral mesoderm (Poulson, 1950), which includes the presumptive musculature. It is therefore probable that $h d p^{2}$ has its primary and perhaps only effect in the musculature. Since the nervous system does not correspond to the same part of the fate-map (Poulson, 1950; Kankel \& Hall, 1976) the muscle degeneration caused by heldup is probably not of nervous origin.

As the defect is probably intrinsic to the muscles, abnormality of the muscles can probably be taken as an indication of their genotype. The assumption that the mutation heldup ${ }^{2}$ can be used as a genetic marker for the muscles permits one to draw several conclusions from these results.

In mosaic flies grossly abnormal fibres can be found adjacent to, and in contact with, fibres that are apparently normal. This indicates that the indirect flight muscles are not derived from a central mobile pool of muscle precursor cells and that there is little mixing of the muscle cells during development. Each muscle precursor cell therefore probably gives rise to a group of cells that remain close together during development; this group probably contributes to one or a small number of fibres only. Since many mosaic fibres are found, 
more than one cell and its progeny contribute to the formation of each fibre, i.e. the nuclei within a fibre are not necessarily clonally related.

The muscles on one side of the mosaic flies were in many cases $(18 \%$ of the total number of sides) themselves mosaic; one or more fibres were frequently absent or abnormal while the others were normal. This suggests that the malefemale boundary in gynandromorphs frequently runs through the foci for muscle abnormality, indicating that they are large.

In quite a large proportion of mosaics (30 out of 357 flies, i.e. $8.4 \%$ ) all of the fibres on one side of the thorax are grossly abnormal or absent, while those on the other side appear completely normal. This suggests that the muscle precursor cells that give rise to the indirect flight muscle do not lie along the ventral midline but are separated from it by some other cells, or that there is some restriction in cells crossing the midline during development.

Since the TDT maps quite far from the indirect flight muscles, the cells that give rise to the TDTs probably do not come from the same pool as those forming the fibrillar muscles.

The number of cells initially present that give rise to any structure can be estimated by using the minimum size of a mutant patch within that structure found in mosaic flies (Stern, 1940; Garcia-Bellido \& Merriam, 1969). Since muscle fibres are syncytial it is not possible to establish the smallest possible size of a mutant patch by the criteria described above; the smallest patch found was one fibre. Since there are 26 indirect flight muscle fibres one obtains a minimum estimate for the number of precursor cells of 26 .

\section{(c) Fate-mapping of behaviour and external morphology of heldup ${ }^{2}$}

The data obtained on the behaviour and the external morphology of $h d p^{2}$ mosaics, which were obtained on the same flies as those sectioned for scoring of the muscles, were also incorporated into the fate-maps (Figs. 6, 7). When intermediate behaviours were observed, these were given values of half different from the cuticular landmarks used. These all map in the same area as the muscles supporting the conclusion that the effects of this mutation are restricted to the muscles. The focus for flightlessness maps in the indirect flight muscles, while the focus for the inability to jump maps close to the TDT. These positions are to be expected, since the indirect flight muscles and the TDT are required for flight and jumping respectively (Behrendt, 1940; Pringle, 1957). Both of these behaviours fit the model of a 'domineering' mutant focus: if the muscles on one side are absent or non-functional the mosaic fly is unable to fly or to jump. The folding of the mesothoracic legs observed in mutant flies upon excitation was too variable a behaviour to be mapped. All the mosaics in which it was observed held their wings vertically, indicating that the foci for leg-folding behaviour are close to those for abnormal wing position. In several mosaic flies only one of the mesothoracic legs was folded; in all such cases the TDT corresponding to the folded leg was extremely abnormal or absent. However, in many flies in 
which the TDTs were abnormal no folding of the mesothoracic legs was observed. This behaviour may therefore be associated with the TDT but be a case of incomplete penetrance.

The leg extension response by $h d p^{2}$ flies to ether was also strongly correlated with abnormal wing position. It was not used in the fate-mapping because many C-S flies also showed the response when exposed to ether for slightly longer periods, and the etherization dose applied proved very difficult to control.

In mosaics of heldup ${ }^{2}$, both wings are held in the same position, whatever that position is. Only six of the $364 h d p^{2}$ mosaics had their wings in different positions, i.e. one held vertically and one held in the normal position. This is probably because the indirect flight muscles as a whole control the shape of the thoracic box and hence the position of the wings (Pringle, 1957), so that the wings act for the most part as a unit. As a result, wing position cannot be analysed by assuming that the positions of the two wings are independent, but must be treated as a 'behaviour' that is produced by the interaction of two bilaterally symmetrical foci, i.e. the position of the foci should be calculated using the 'submissive' or the 'domineering' models. When this is done the abnormal wing position maps in the 'muscle' region of the blastoderm (Hotta \& Benzer, 1972; Fig. 6); more specifically, it corresponds to that area in which the indirect flight muscles map, as expected from the role of these muscles in controlling the shape of the thoracic box.

The sharp bend in the femur observed occasionally in mutant flies is seen at a much higher frequency in mosaic flies. The fate-map focus obtained for this characteristic is unusual in that the bending seems to be closely correlated to both the muscles and the leg cuticle; i.e. the bending is only observed, with few exceptions, if the muscle is abnormal and the cuticle is yellow, but is not observed if only one of these conditions is true. This suggests that leg bending is associated with cuticular weakness; this could be due to expression of the $h d p^{2}$ mutation in the cuticle or to a cuticular defect associated with the marker yellow. The latter is probably true since a high proportion ( $90 \%$ ) of $y h d p^{2}$ flies show femoral bending while few $(5 \%) h d p^{2}$ flies have bent femurs.

\section{(B) The mutation upheld (up)}

The mutation upheld was isolated and mapped to 1-41 by Fahmy (Lindsley \& Grell, 1968), who kindly supplied me with mutant flies. Another allele of this gene was isolated and studied by Hotta \& Benzer (1972) who referred to it as wingsup $B$. This second allele will be referred to as upheld ${ }^{2}\left(u p^{2}\right)$ in this paper. Upheld flies hold their wings vertically; penetrance of this phenotype is $100 \%$ in males and $95 \%$ in females for both alleles. The wing position of up flies is dependent on the temperature at which the flies are raised. Flies raised at $29^{\circ} \mathrm{C}$ all held their wings vertically, while many $(60 \%)$ of those raised at $18{ }^{\circ} \mathrm{C}$ held their wings in a ventrolateral position similar to that of flapwing (see below). However, all up flies were flightless and had grossly abnormal muscles regardless 


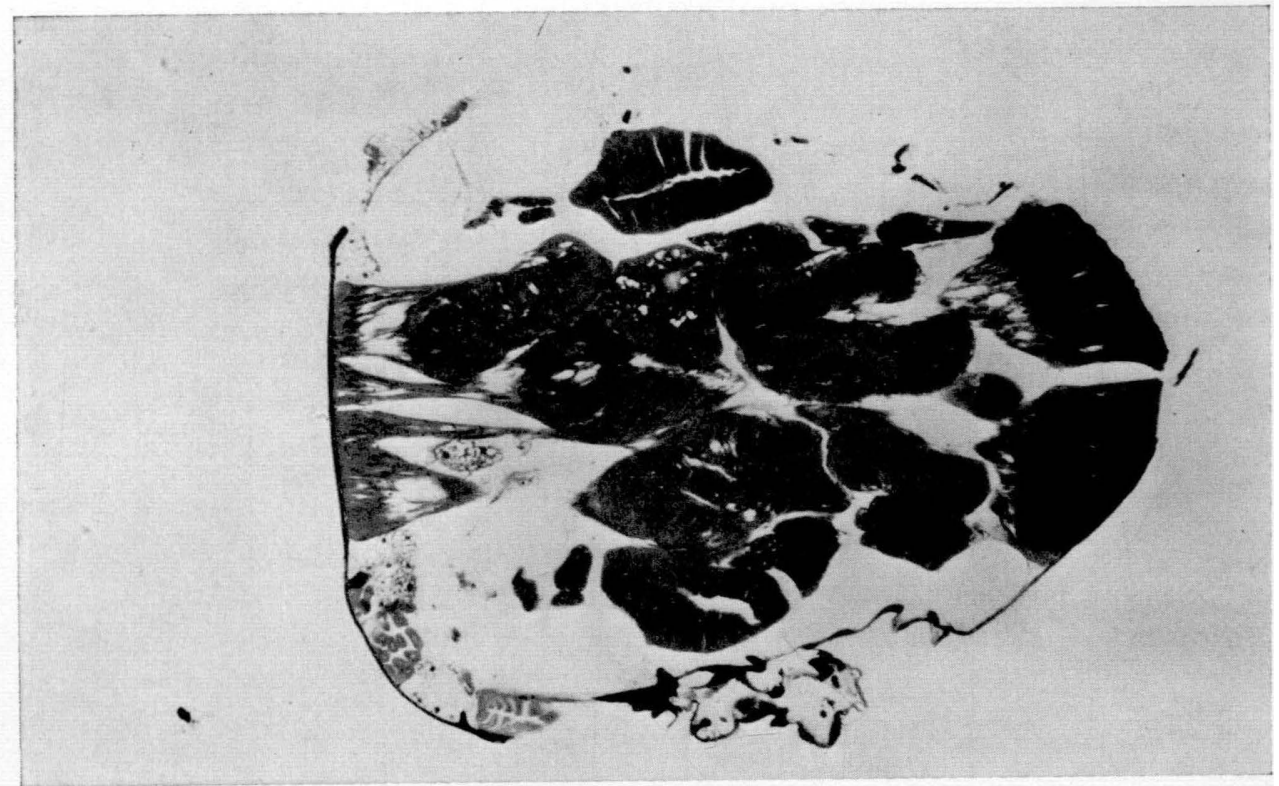

Fig. 8. Longitudinal section through the thorax of an upheld fly. Specimen silverstained and sectioned in plastic at $2 \mu \mathrm{m} . \times 100$.

of the temperature at which the flies had developed. No effect of temperature on $u p^{2}$ flies was observed. Viability of $u p$ is $85 \%$ and of $u p^{2} 60 \%$. All upheld flies are flightless and unable to jump; $u p /+$ females are also flightless, although their wings are held in the normal position, and jump poorly. In some flies the mesothoracic legs are folded under the thorax after recovery from etherization; this effect is more pronounced in $u p^{2}$ flies than in $u p$ flies.

In both alleles, much of the flight musculature is present but grossly abnormal (Fig. 8). In $u p^{2}$ the flight muscles lack cross-striations (Hotta \& Benzer, 1972); other muscle structures such as thick and thin filaments, and mitochondria, are present, although not arranged in an organized manner. Females heterozygous for the mutation have a normal complement of well-organized fibres that contain all of the muscle components but lack $50 \%$ of the Z-band material (Hotta, personal communication). The allele up has less effect on the musculature than $u p^{2}$ : more muscle tissue is present in the former, and some crossstriations can be seen. In both mutants, as in heldup, the anterior part of the flight muscles is more severely affected than the posterior part; the posterior DVMs being least affected. The TDTs are generally absent or very small in $u p^{2}$; in $u p$ they are usually present, although abnormal and reduced in size.

Unlike heldup, $u p$ and $u p^{2}$ affect the development of the flight muscles, and do not cause degeneration; at no time during pupal development is the muscle present in normal quantities or with normal morphology (Hotta \& Benzer, 1972). 


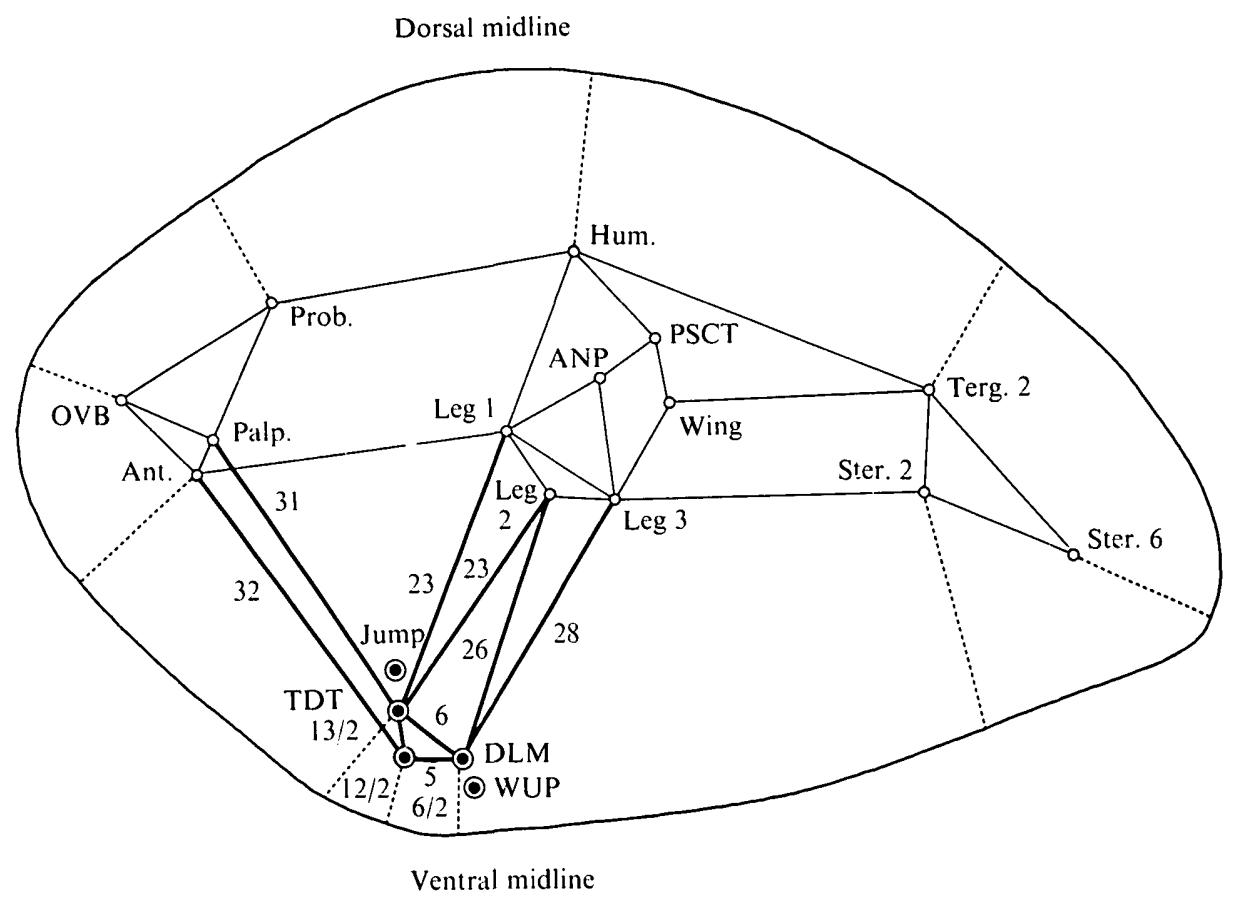

Fig. 9. Fate-map of the behavioural and anatomical defects of the mutant upheld ${ }^{2}$.

Numbering and lettering as in Fig. 6.

Three hundred and two flies mosaic for $u p^{2}$, and 106 mosaic for $u p$ were produced and examined. As it was difficult to identify each fibre unambiguously, because the mutation causes the fibres to be very variable in thickness and to be very distorted, fibres were scored in groups rather than individually. The fibres were scored as normal if no abnormality was detectable, as abnormal if absent or grossly abnormal, and intermediate if slightly abnormal. No histochemical defect was found in these muscles, so only morphological criteria could be used for the scoring.

The groups scored were the DLMs, the anterior DVMs and the TDTs. The posterior DVMs could not be reliably scored because in many flies they were indistinguishable from wild-type fibres with the light microscope; electron microscopy showed, however, that these fibres had an ultrastructure typical of mutant fibres. For the fate-map calculations, fibres scored as intermediate were given a value of half-different in genotype from all other landmarks.

The fate-map of the behavioural and morphological foci of $u p^{2}$ (Fig. 9), and of up are essentially the same as that obtained for heldup (Fig. 6) and confirm the conclusions drawn from the study of that mutant, and the results of Hotta $\&$ Benzer (1972) for $u p^{2}$ (wingsup B). The gene upheld therefore probably also has its site of action in the musculature. 


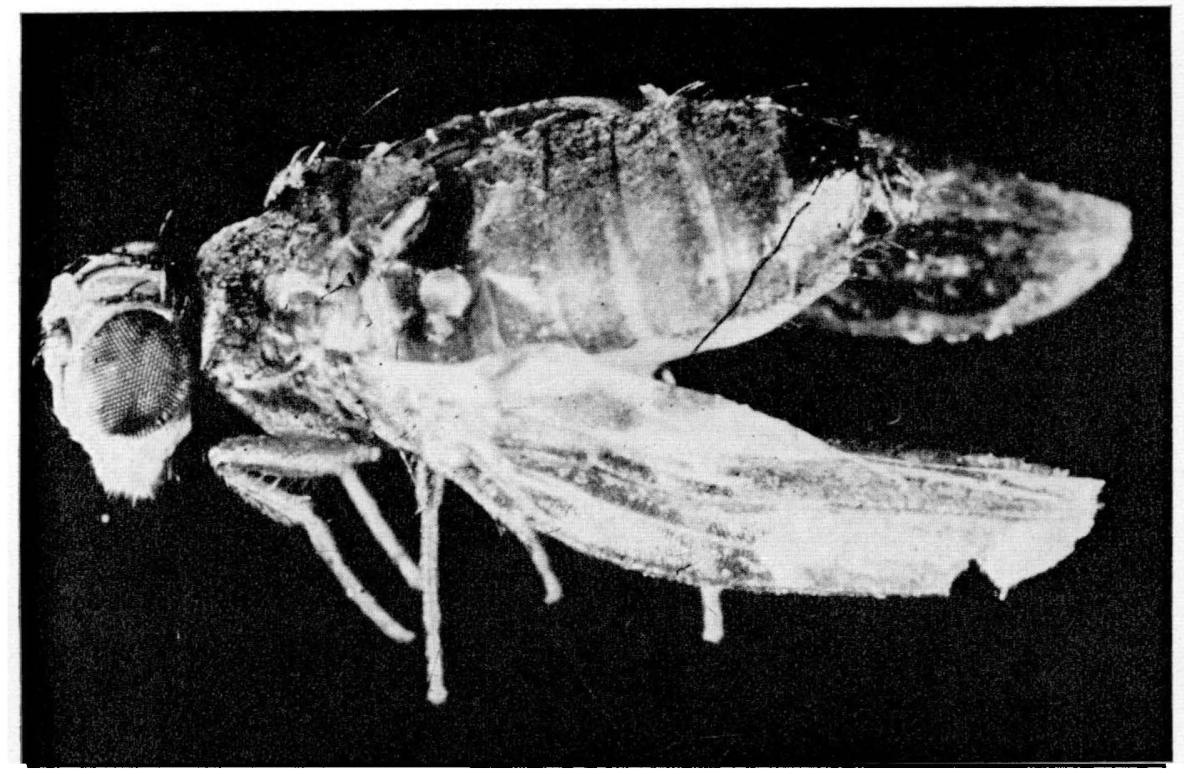

Fig. 10. Lateral view of an int fly. Note the deep anterior indentation of the thorax. The wings are abnormal due to damage due to being trailed while walking. $\times 30$.

\section{(C) The mutation indented thorax (int)}

This mutation was isolated by Dr A. Ghysen at the California Institute of Technology by EMS mutagenesis of flies carrying the inversion $\operatorname{In}(l) y^{4}, y^{4}$, from which the mutation was removed by outcrossing to C-S flies. Preliminary mapping places the mutation equidistant from $c v$ and $v$, i.e. at about 1-20 to $1-25.80-85 \%$ of int flies hold their wings ventrolaterally, $10 \%$ hold them vertically, and the remainder have them in the normal position.

In many flies the dorsal thorax is indented (Fig. 10); the position and extent of this indentation is variable. The indentations are observed in a higher proportion $(85 \%)$ of flies raised at $29^{\circ} \mathrm{C}$ than of flies raised at $18{ }^{\circ} \mathrm{C}(45 \%)$, and are more pronounced in the former. They are also more pronounced in $\operatorname{In}(1) y^{4}$, $y^{4}$ int flies; this may be because the cuticle is weaker if yellow. In flies homozygous for $y$, int is partially dominant: $30 \%$ of $\operatorname{In}(1) y^{4}, y^{4}$ int $/ y$ flies are flightless and some have small thoracic indentations.

The penetrance for flightlessness and for inability to jump of int flies is $100 \%$ regardless of the temperature at which the flies were raised.

(D) The mutation flapwing (flw)

The thorax contains the normal complement of muscles, and these appear normal at the level of the light microscope except that when the thorax is indented some of the indirect flight muscle fibres are displaced by the indented cuticle. Preliminary electron-microscopic examination shows that, although the 


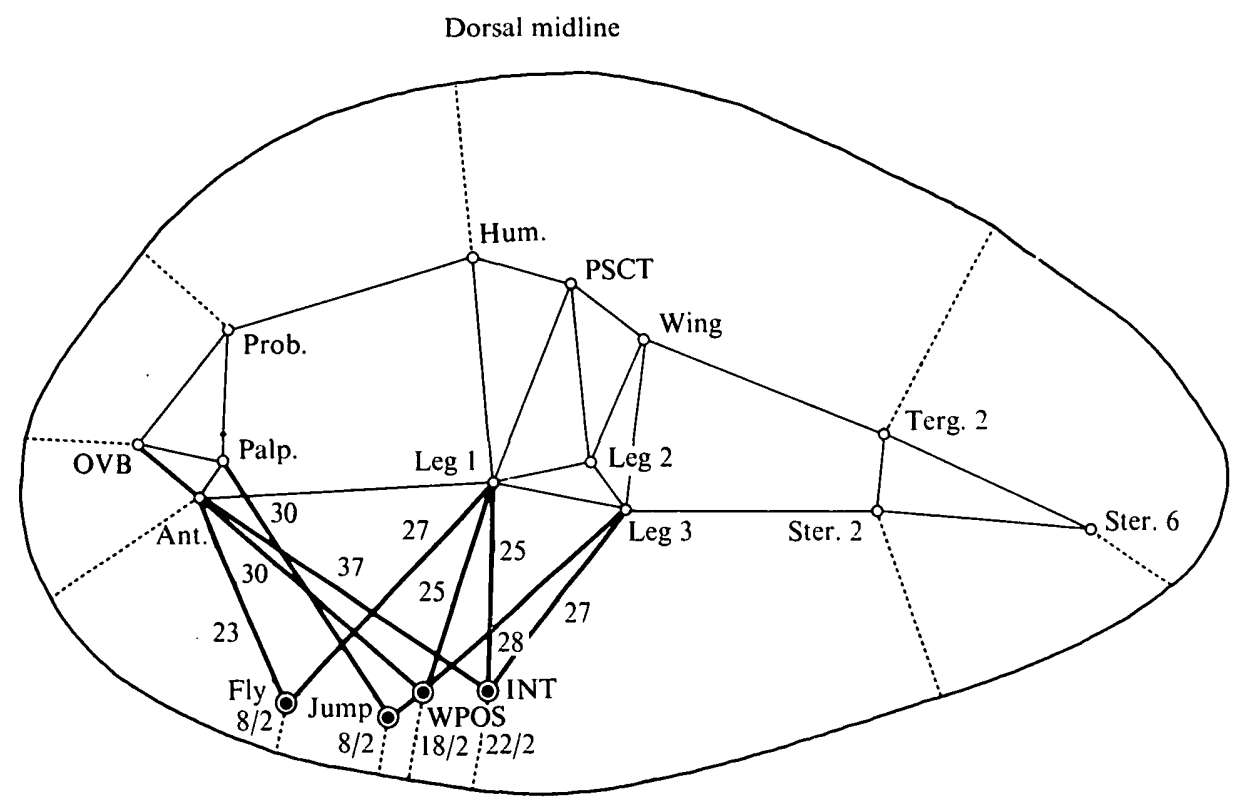

Ventral midline

Fig. 11. Fate-map of the behavioural defects of the mutant indented thorax. Numbering and lettering as in Fig. 6. WPOS refers to abnormal wing position.

thorax contains the normal complement of muscles, the structure of the myofibrils in the indirect flight muscle fibres is abnormal.

One hundred and seventy-eight flies mosaic for int were examined with respect to the shape of their thoraces, their wing position, and ability to fly and jump. The muscles were not examined since they could not be distinguished from normal muscles in the light microscope. The fate-map for int behaviour (Fig. 11) is similar to those previously described: all mutant characteristics map in that area of the blastoderm that corresponds to the ventral mesoderm, indicating that this mutant, like the others, has its primary effect in the musculature.

The mutant foci for flightlessness and inability to jump were 'domineering', while those for wing position and for thoracic indentation were 'submissive' (Table 1). These results suggest that the flies cannot fly or jump if half of their flight or jump musculature is defective, while more of the musculature must be abnormal to produce thoracic indentation and hence the abnormal wing position characteristic of this mutant. In several of the mosaics the wing position and thoracic indentation were intermediate: one side of the thorax was indented, and only the corresponding wing held down. These two abnormalities can be treated as if the two foci were independent, and mapped accordingly: the foci lie very close to those found treating the foci as 'submissive'. 

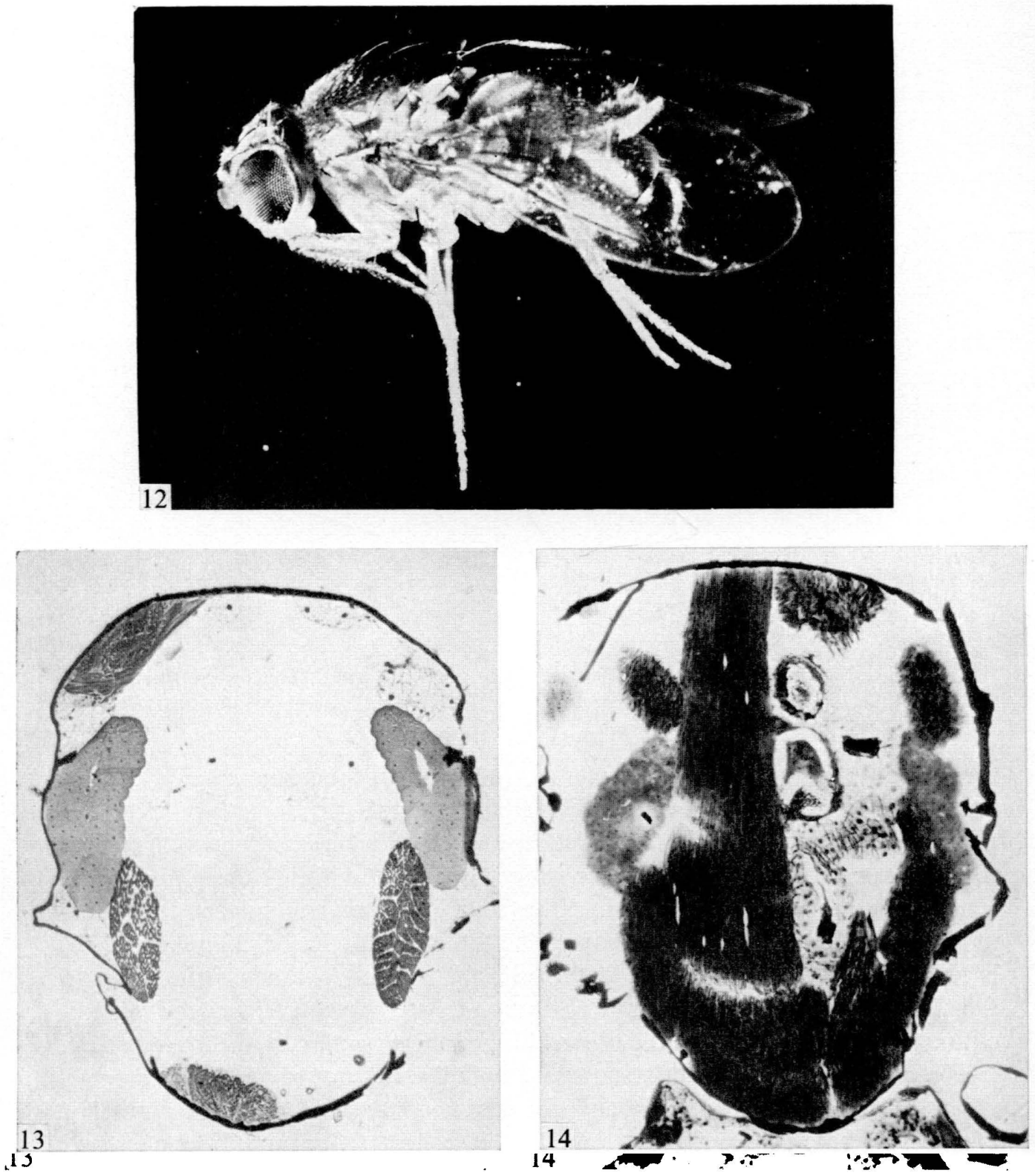

Fig. 12. Lateral view of a flapwing ${ }^{2}$ fly. $\times 30$.

Fig. 13. Longitudinal section through the thorax of a $f w^{2}$ fly. Specimen Bielchowsky silver-stained and sectioned at $2 \mu \mathrm{m} . \times 100$.

Fig. 14. Longitudinal section through the thorax of a mosaic $y c h o f\left(w^{2} / \operatorname{In}(1) w^{\mathrm{vC}}\right.$ fly. Fly frozen-sectioned and silver-stained. The left side is largely normal, although two anterior dorsoventral fibres are missing, while most of the fibres on the right side are absent or abnormal. $\times 110$. 
Table 1. Data from int mosaics

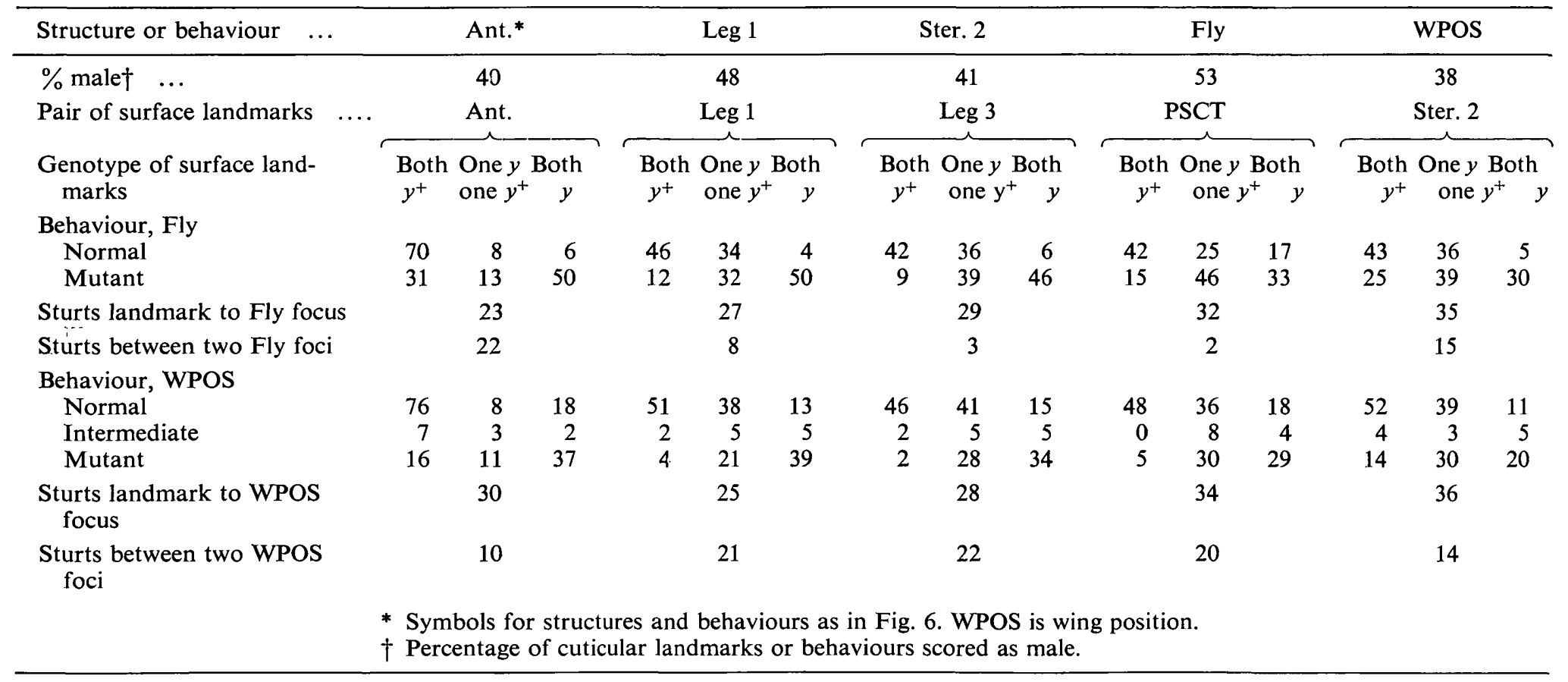


Table 2. Data from flw $^{2}$ mosaics

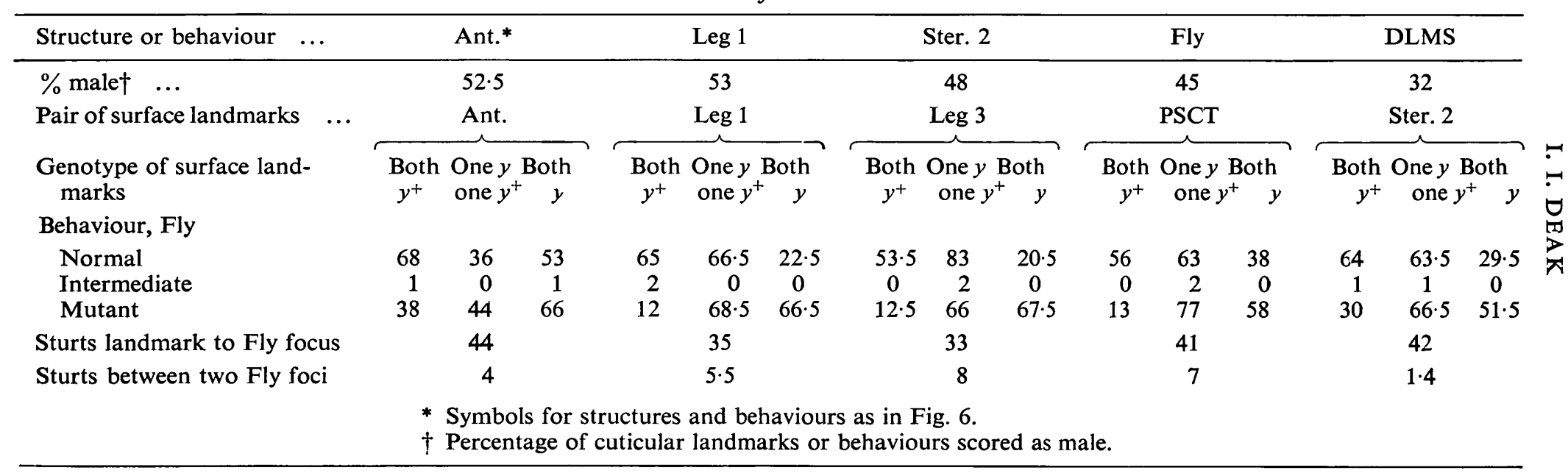


One allele of this gene was obtained from the stock collection of the California Institute of Technology; a second was recently isolated by Dr W. Hanratty of the University of Pittsburgh, who kindly supplied me with the mutant flies; no differences between the two alleles were found. All of the data presented below were obtained using the latter $\left(f l w^{2}\right)$. The mutation $f w$ is recessive and maps at 1-31 (Lindsley \& Grell, 1968); it has a viability of $70 \%$. The penetrance for flightlessness is $100 \%$; about $70 \%$ of the flies can make very small jumps of less than $1 \mathrm{~cm}$, while the remaining $30 \%$ are unable to jump. In $90-95 \%$ of the flies the wings are held ventrolaterally (Fig. 12) at an angle ranging from $5^{\circ}$ to $30^{\circ}$ ventrally and $5^{\circ}$ to $60^{\circ}$ laterally (the greater the ventral displacement the greater the lateral); these angles increase during the first few days after emergence. In some flies in which the ventrolateral displacement is small, excitation results in the wings being raised into the vertical position; within a few minutes they return to their depressed state. Up to $5 \%$ of the flies hold their wings vertically as a result of such excitation, while in about $5 \%$ of the flies the wings are permanently held in the vertical position. In $2-3 \%$ of the flies the wing position is normal.

The mutation also causes some cuticular defects: in many flies the posterior cross-vein is incomplete, the anterior scutellar bristles are missing, and the eyes are slightly roughened.

Figure 13 shows a horizontal section through the thorax of a $f w^{2}$ fly. The ventralmost DLMs (1-4) and the anterior DVMs (7-9) are absent in all flies. In the $2-3 \%$ of flies that have their wings in the normal position the DLMs 5 and 6 are present; these may suffice to keep the thoracic box the normal shape. - In most of the flies one to four of the posterior DVMs (10-13) are present; about $10 \%$ of the flies contain no indirect flight muscle fibres at all. There is no apparent correlation between the ventrolateral wide angle and the number of fibres present.

The TDTs appear normal by light microscopy, although the poor jumping ability of these flies may indicate that these muscles are also abnormal. In some mutant flies structures resembling larval fat-body cells were found in the thorax.

Three hundred and seven flies mosaic with respect to $f w^{2}$ were produced and sectioned. In individuals that lacked the fibres on one side of the thorax the fibres on the other side undergo hypertrophy, pushing the cardia and the thoracic ventriculus into the empty half of the thorax (Fig. 14). Each fibre was scored by its presence or absence alone: no intermediate fibres were found.

The data obtained from $f w^{2}$ mosaics indicate that the behavioural foci for flight and jump are 'submissive', and that only $32 \%$ of the muscles were scored as mutant (Table 2).

The fate-map obtained for $f w^{2}$ indicates that the foci of this mutation also fall in the ventral mesoderm (Fig. 15), which may indicate that the site of action of this mutation is in the musculature. However, since the foci for this mutant are 'submissive' and are displaced somewhat posteriorly relative to those of 


\section{Dorsal midline}

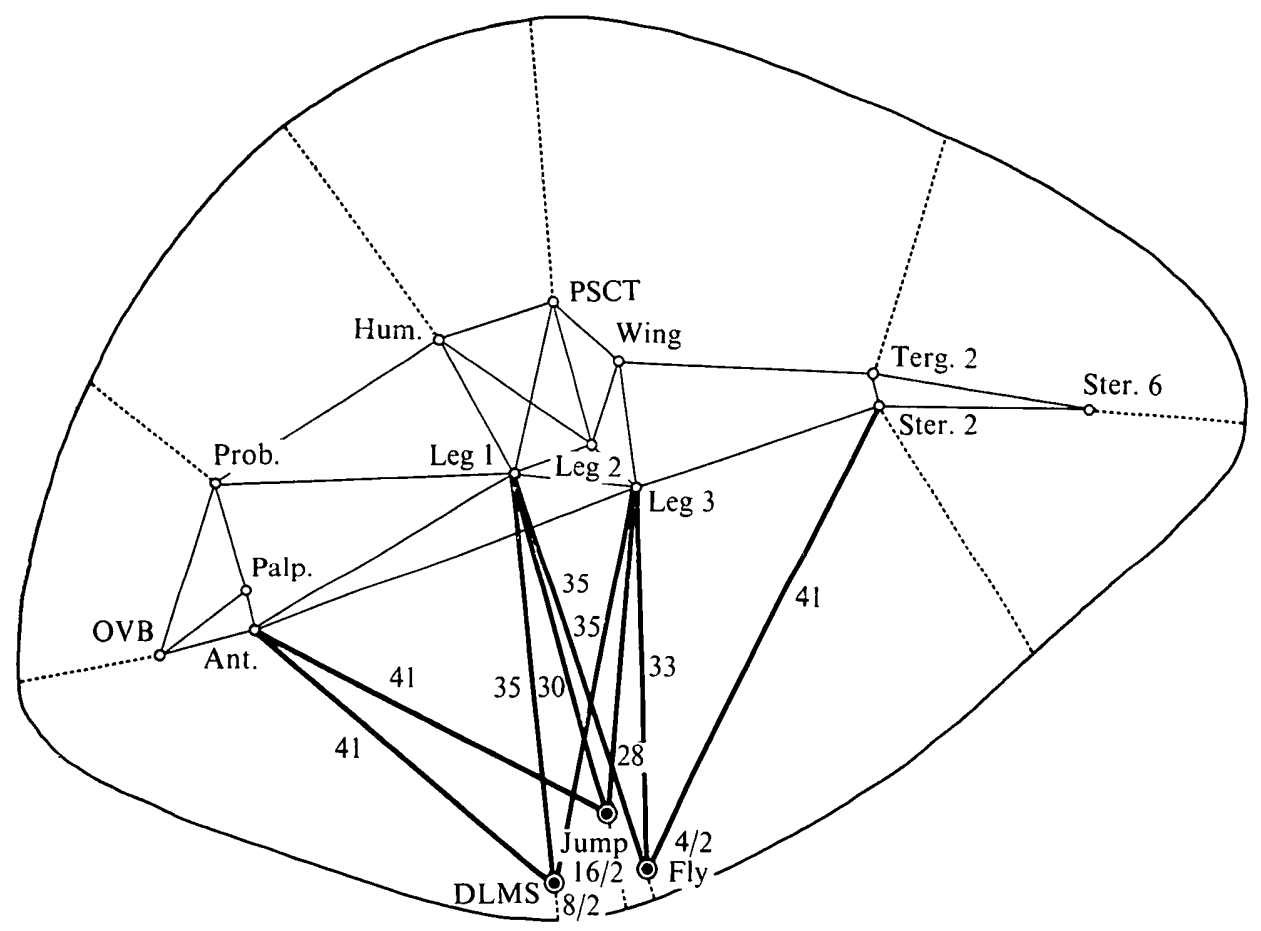

Ventral midline

Fig. 15. Fate-map of the behaviour and anatomical defects of the mutant flapwing ${ }^{2}$. Numbering and lettering as in Fig. 6.

the 'muscle mutants' (compare with Figs. 6, 9, and 11) it is possible that a different mesodermal structure is affected by the mutation $f w$.

The fat-body has been postulated to derive from this area on the fate-map (Falk, Orevi \& Menzl, 1973; Nissani, 1975; Janning, 1976) and has been shown by histological techniques to derive from the corresponding part of the ventral mesoderm (Sonnenblick, 1950).

The fat-body could be implicated in muscle development in several ways. It could release factors essential for muscle formation into the haemolymph. A mutation resulting in the absence of such factors would prevent muscle development, and its behavioural foci could well be 'submissive', since release of the factor(s) from one half of the fat-body could be sufficient to permit normal development of the muscles. It would, however, be difficult to explain the absence of individual fibres in $f l w$ mosaics if the gene acts by release of a diffusible compound, unless its diffusion were in some way restricted. Alternatively, fat-body cells, which are released into the haemocoele during early pupation, may be involved in the provision of food to the developing muscles (Whitten, 


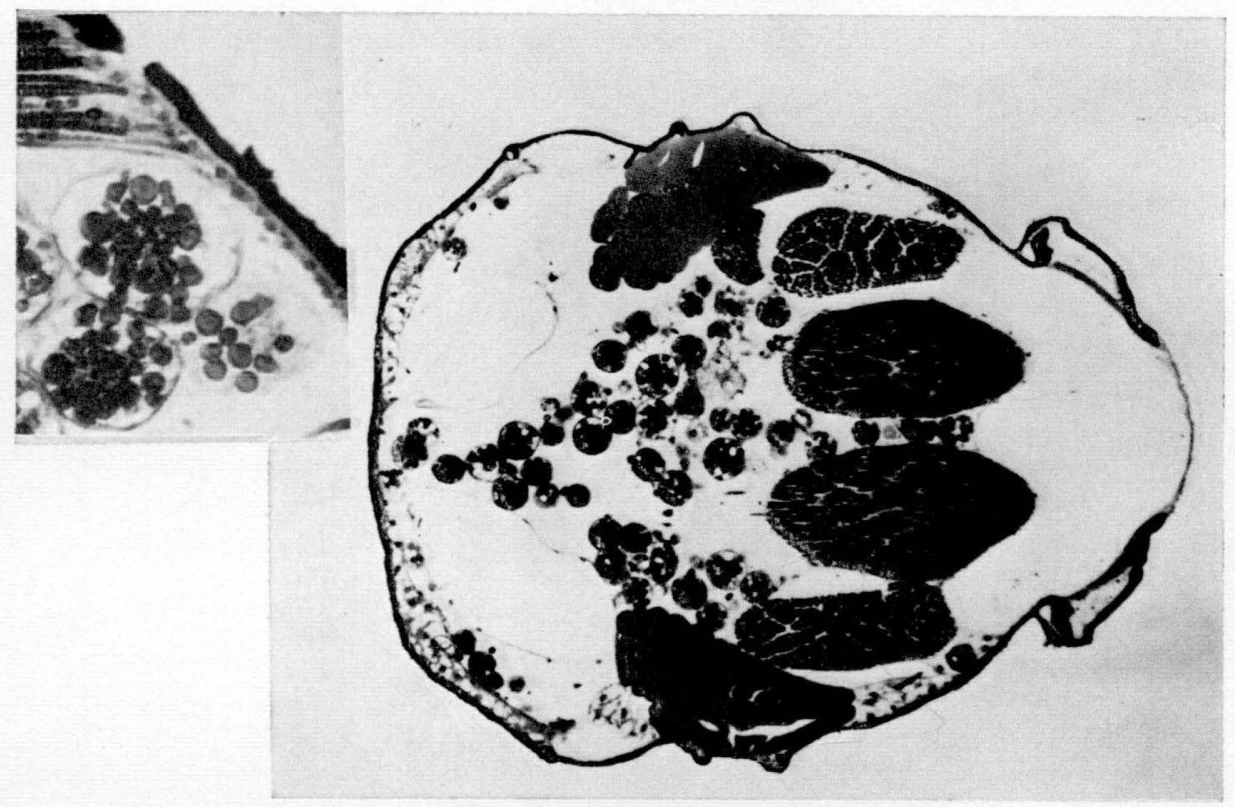

Fig. 16. Longitudinal section through the thorax of a vertical wing fly. Specimen Bielchowsky silver-stained and sectioned at $2 \mu \mathrm{m}$. The majority of the indirect flight muscle is absent. Note also the presence of the spherical bodies referred to in the text. $\times 90$. The inset shows these bodies at higher magnification. $\times 400$.

1962, 1964). However, fat-body cells are thought to be stationary (Whitten, 1964); one could then explain the presence of individual defective fibres, but such a model would result in behaviour being 'domineering', and in $50 \%$ of the muscle being abnormal.

A hypothesis that could explain the results presented here would be that fat-body cells after release move to a limited extent before settling at a particular site to supply the developing muscles, and that the mutation $f w$ results in the production and release of defective fat-body cells. In mosaic animals the normal fat-body cells would by virtue of their limited mobility be able to compensate partially for the lack of functional fat-body cells in the mutant part. The fatbody cell-like structures seen occasionally in the thoraces of adult $f l w$ flies may be the defective fat-body cells which have been unable to deliver their contents to the developing muscle.

Fat-body cells may also contribute to development of the thoracic tracheal air sacs (Whitten, 1964). The presence of bodies resembling fat-body cells in vertical wing flies (see below; Fig. 16) may be of interest in this respect.

The gene $f w^{+}$also functions in the cuticle, since the cuticular abnormalities that are observed correlate with the cuticular genotype. 
Dorsal midline

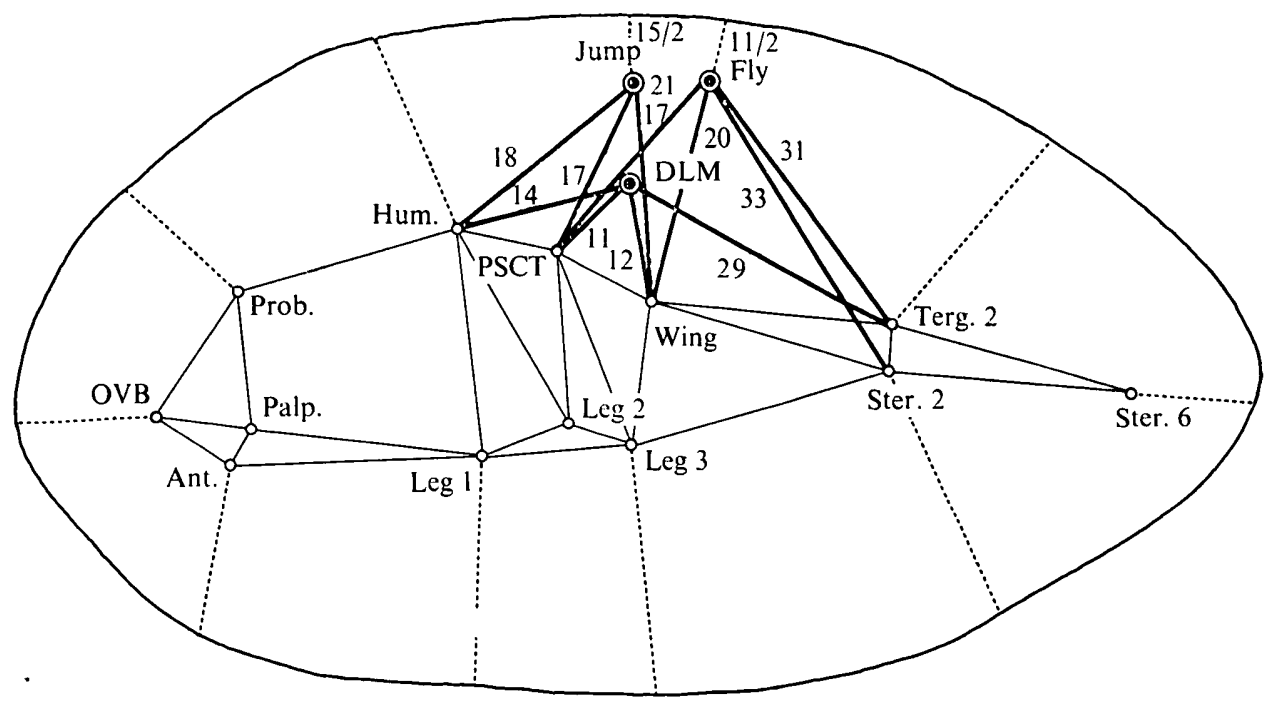

Ventral midline

Fig. 17. Fate-map of the behavioural and anatomical defects of the mutant vertical wing. Numbering and lettering as in Fig. 6.

(E) The mutation vertical wings (vtw)

Two alleles of this gene were isolated by Barbara Butler at the California Institute of Technology by EMS mutagenesis of C-S flies. Both alleles are recessive, have a viability of $95 \%$, and map between $c v$ and $s n$, nearer $s n$, i.e. at about 1-18. Both mutants are flightless (penetrance $100 \%$ for $v t w, 85 \%$ for $\left.v t w^{2}\right)$ and cannot jump more than $2 \mathrm{~cm}$. They hold their wings vertically (penetrance of $v t w 90 \%$, of $v t w^{2} 63 \%$ ). In $v t w$ flies minor disturbances of the cuticular structure just anterior to the distal portion of the second wing vein can be seen; these were not found in $v t w^{2}$ flies.

All $v t w$ flies lack the DLMs 1-4 and the anterior DVMs 7-9; in about $8 \%$ of the flies ( $80 \%$ of which hold their wings in the normal position) fibres 5 and 6 are present, and in $30-40 \%$ of flies one to four of the posterior DVMs 10-13 are present (Fig. 16). In $v t w^{2}$ the pattern is similar but many more fibres are present: fibres 5 and 6 (and occasionally fibres 1-4) and 10-13 are present in $60-70 \%$ of the flies.

One type of structure is seen in the thorax of adult $v t w$ flies that is normally only found in early pupae of C-S flies, namely spherical bodies containing 20-40 smaller spheres (Fig. 16). These bodies appear similar to fat-body cells, which 
Table 3. Data for vtw mosaics

\begin{tabular}{|c|c|c|c|c|c|c|c|c|c|c|c|c|c|c|c|c|}
\hline $\begin{array}{c}\text { Structure or } \\
\text { behaviour } \quad \ldots\end{array}$ & \multicolumn{3}{|c|}{ Ant.* } & \multicolumn{3}{|c|}{ Leg 1} & \multicolumn{3}{|c|}{ Ster. 2} & \multicolumn{3}{|c|}{ PSCT } & \multicolumn{2}{|r|}{ Fly } & \multicolumn{2}{|r|}{ DLMs } \\
\hline$\%$ male $\dagger \quad \ldots$ & \multicolumn{3}{|c|}{55} & \multicolumn{3}{|c|}{57} & \multicolumn{3}{|c|}{51} & \multicolumn{3}{|c|}{60} & \multicolumn{2}{|r|}{70} & \multicolumn{2}{|r|}{51} \\
\hline $\begin{array}{l}\text { Pair of surface land- } \\
\text { marks }\end{array}$ & \multicolumn{3}{|c|}{ Prob. } & \multicolumn{3}{|c|}{ Hum. } & \multicolumn{3}{|c|}{ PSCT } & \multicolumn{3}{|c|}{ Wing } & \multicolumn{2}{|r|}{ Leg 3} & \multicolumn{2}{|r|}{ Ster. 2} \\
\hline $\begin{array}{l}\text { Genotype of surface } \\
\text { landmarks }\end{array}$ & $\begin{array}{c}\text { Both } \\
y^{+}\end{array}$ & $\begin{array}{l}\text { One } y \\
\text { one } y^{+}\end{array}$ & Both & $\begin{array}{c}\text { Both } \\
y^{+}\end{array}$ & $\begin{array}{l}\text { One } y \\
\text { one } y^{+}\end{array}$ & $\begin{array}{l}\text { Both } \\
+\quad y\end{array}$ & $\begin{array}{c}\text { Both } \\
y^{+}\end{array}$ & $\begin{array}{l}\text { One } y \text { I } \\
\text { one } y^{+}\end{array}$ & $\begin{array}{l}\text { Both } \\
+\quad y\end{array}$ & $\begin{array}{c}\text { Both } \\
y^{+}\end{array}$ & $\begin{array}{l}\text { One } y \\
\text { one } y^{+}\end{array}$ & $\begin{array}{l}\text { Both } \\
+\quad y\end{array}$ & $\begin{array}{c}\text { Both } \\
y^{+}\end{array}$ & $\begin{array}{l}\text { One } y \text { Both } \\
\text { one } y^{+} \quad y\end{array}$ & $\begin{array}{r}\text { Both } \\
y^{+}\end{array}$ & $\begin{array}{l}\text { One } y \text { Both } \\
\text { one } y^{+} y\end{array}$ \\
\hline \multicolumn{17}{|l|}{ Behaviour, Fly } \\
\hline $\begin{array}{l}\text { Normal } \\
\text { Mutant }\end{array}$ & $\begin{array}{l}35 \\
22\end{array}$ & $\begin{array}{l}19 \cdot 5 \\
19 \cdot 5\end{array}$ & $\begin{array}{l}11 \cdot 5 \\
70 \cdot 5\end{array}$ & $\begin{array}{l}36 \cdot 5 \\
11 \cdot 5\end{array}$ & $\begin{array}{l}25 \cdot 5 \\
34 \cdot 5\end{array}$ & $\begin{array}{r}4 \\
66\end{array}$ & $\begin{array}{r}32 \\
2\end{array}$ & $\begin{array}{l}30 \\
41\end{array}$ & $\begin{array}{r}4 \\
69\end{array}$ & $\begin{array}{r}33 \\
2\end{array}$ & $\begin{array}{l}29 \cdot 5 \\
40 \cdot 5\end{array}$ & $\begin{array}{r}2 \cdot 5 \\
68 \cdot 5\end{array}$ & $\begin{array}{l}31 \cdot 5 \\
10 \cdot 5\end{array}$ & $\begin{array}{l}31 \cdot 5 \\
34 \cdot 5\end{array}$ & $\begin{array}{l}29 \\
21\end{array}$ & $\begin{array}{rr}29 \cdot 5 & 7 \cdot 5 \\
43 \cdot 5 & 47 \cdot 5\end{array}$ \\
\hline $\begin{array}{l}\text { Sturts landmark to } \\
\text { Fly focus }\end{array}$ & & 31 & & & 24 & & & 21 & & & 20 & & & 27 & & 33 \\
\hline \multirow{2}{*}{$\begin{array}{l}\text { Sturts between two } \\
\text { Fly foci }\end{array}$} & & 19 & & & 16 & & & 7 & & & 8 & & & 14 & & 24 \\
\hline & & & $\begin{array}{l}* \mathrm{Sy} \\
\dagger \mathrm{Pe}\end{array}$ & $\begin{array}{l}\text { mbols } \\
\text { centag }\end{array}$ & $\begin{array}{l}\text { for struc } \\
\text { ge of cut }\end{array}$ & $\begin{array}{l}\text { Ictures } \\
\text { iticula }\end{array}$ & $\begin{array}{l}\text { and be } \\
\text { landm }\end{array}$ & $\begin{array}{l}\text { haviours } \\
\text { arks or }\end{array}$ & $\begin{array}{l}\text { rs as ir } \\
\text { behav }\end{array}$ & $\begin{array}{l}\text { Fig. } 6 . \\
\text { ours sc }\end{array}$ & cored as & male. & & & & \\
\hline
\end{tabular}


detach from the fat-body early during pupation (Whitten, 1962, 1964), and may provide food to the developing muscles and tracheae. They may also be sarcolytes, fragments of degenerating muscle tissue produced by the histolysis of larval muscles.

The effect of the mutation $v t w$ is not to cause degeneration of the muscles but to block development, since at no time during development are normal indirect flight muscles seen.

All the fibres that remain in vertical wing flies are indistinguishable in the light microscope from normal fibres; in the electron microscope most of the fibrillar structure is normal, but the fibres occasionally contain membrane inclusions.

One hundred and seventy-eight flies mosaic for $y$ cho vtw were examined. Mosaics of this mutation differ from those of the other mutations in two respects. First, the muscle fibres on one side of the animal were, in almost all cases $(97 \%)$, either all present or all absent. This suggests that the male-female boundary line in gynandromorphs rarely runs through the foci for muscle abnormality, indicating that they are small. Second, the muscles on the two sides were much more often of different phenotype than in mosaics of the other mutants, suggesting that the $v t w$ foci lie much further from the midline than those of the other mutants. Indeed, the data given in Table 3, and the fate-map (Fig. 17) derived from $v t w$ mosaics, show that this gene acts at a very different site from the other mutants (see Figs. 6, 9, 11, and 15, for comparison). The foci for defective muscles and for the inability to fly and to jump (both of which behaviours are 'domineering') map dorsal to the thoracic cuticle. The larval tracheae also map dorsal to the thoracic cuticle (Poulson, 1950; Janning, 1975); it is not known whether adult tracheae map in this area. This suggests that the mutation $v t w$ has its primary effect in the tracheae, or, less probably, in the dorsal thoracic cuticle, but definitely not in the ventral mesoderm. The gene $v t w$ is also expressed in the cuticle: the blemishes observed adjacent to the second wing vein are present in mosaic flies only when the wing cuticle is of mutant genotype.

\section{DISCUSSION}

Each of the five $X$-linked genes (eight mutations) described above has a different role in the development of muscle. Two are involved in the formation of muscle tissue; one may be associated with the fat-body (flapwing) and the other may act in the tracheae (vertical wing); a third is required more for the organization of the muscle than for its formation (upheld); another is involved in the maintenance of muscle structure once formed (heldup); and the fifth seems to be required for the detailed architecture of the muscle fibre (indented thorax).

In those mutations that cause a reduction in the quantity of the flight muscles (i.e. all except int) the effect of the mutation appears to be most severe in the 
anterior end of the thorax, suggesting that when the necessary gene products are limiting the posterior end of the thorax is preferentially supplied.

With the exception of $f w$, all the mutations have 'domineering' behavioural foci for flightlessness and the inability to jump: the majority of mosaics having bilaterally mosaic thoracic cuticle were unable to fly or jump.

Although the viability of some of the mutants is reduced relative to C-S flies ( $u p^{2} 60 \%, h d p^{2} 65 \%$ ) mosaics of all mutations had approximately $40-60 \%$ of their cuticle male. The exact proportion of maleness varies with the cuticular structure scored; this is also true for $\operatorname{In}(1) w^{r C} / y$ cho mosaics, making it unlikely that the reduced viability of the mutations results in marked distortion of the fate-maps.

In all mutants and in almost all mosaic flies (except for int mosaics) both wings were held in the same position, regardless of what the position was. This is because the indirect flight muscles work indirectly on the thorax to change its shape, and hence the position of the wings (Boettiger \& Furshpan, 1952; Pringle, 1957). In int the thorax itself is distorted so that the relationship of flight muscles to wing position is changed.

All of the mutations affect the indirect flight muscles preferentially (they were selected in such a way as to favour this class of mutants), but none seems to act in this tissue only. All seem to affect the TDTs (in that none of the mutants can jump as well as C-S flies), although only in heldup and upheld is this reflected in gross morphological abnormality. Heldup and upheld also affect the leg muscles, and indirectly the leg cuticle (leg weakness, femoral bending). $f l w$ causes minor cuticular abnormalities in addition to its gross effect on the musculature, and $v t w$, whose probable primary effect on the tracheae is reflected in gross abnormality of the muscles, is also expressed in the cuticle. Thus detailed examination of mutations selected as specific to one tissue shows that these often have effects on other tissues of different embryonic origin. It also shows that effects of mutations may be indirect rather than being due to the gene being expressed in the tissue itself (for example, the thoracic indentation in int flies). Fate-mapping is therefore useful in delineating the target tissue of a mutation and in segregating primary effects on a tissue from secondary phenomena resulting from defective function of some other tissue.

In order to study muscle development in mosaic flies it would be extremely valuable to have a marker for muscle genotype that is independent of any muscle abnormality mutation, whose primary site of action can only be inferred. The conclusions presented above on the development of muscle must remain tentative until it can be definitely shown that the mutations heldup and upheld are primary muscle mutations and that they can be used as markers for the muscle genotype.

If we assume this to be the case, we can tentatively conclude that the indirect flight muscle fibres are not derived from a central mobile pool of precursor cells, and that there is little mixing of cells during development: each precursor 
cell probably gives rise to a group of cells that remain together during development and contribute to a small number of fibres only. Further, since mosaic fibres are found, the nuclei within each fibre are not necessarily clonally related. This conclusion agrees with that of Mintz \& Baker (1967), who used genetic mosaicism in allophenic mice to investigate the origin of syncytial muscle.

This work was done while I. I. Deak was the recipient of a fellowship from the Muscular Dystrophy Association, and was supported by a National Science Foundation grant to Dr Seymour Benzer.

I thank Dr Benzer for his advice and support during my stay at the California Institute of Technology, Barbara Butler, Joyce Gorn and Mike Cousineau for their excellent assistance, Duncan Byers for his invaluable help with the computer programmes, and my many colleagues for their help and criticism throughout the work and throughout the preparation of this manuscript.

\section{REFERENCES}

BEHRENDT, R. (1940). Untersuchungen über die Wirkungen erblichen und nicht-erblichen Fehlens bezw. Nichtgebrauchs der Flügel auf die Flugmuskulatur von Drosophila melanogaster. Z. wiss. Zool. 152, 129-158.

Benzer, S. (1973). Genetic dissection of behaviour. Scientific American 229 no. 6, 24-37.

Boettiger, E. G. \& Furshpan, E. (1952). The mechanics of flight movements in Diptera. Biol. Bull. mar. biol. Lab., Woods Hole 102, 200-211.

DEAK, I. I. (1976a). A histochemical study of the muscles of Drosophila melanogaster. J. Morph. (In the Press.)

DEAK, I. I. (1976b). Use of Drosophila mutants to investigate the effect of disuse on the maintenance of muscle. J. Insect Physiol. 22, 1159-1165.

Falk, R., Orevi, N. \& Menzl, B. (1973). A fate map of larval organs of Drosophila and preblastoderm determination. Nature, Lond. 246, 19-20.

Garcia-Bellido, A. \& Merriam, J. R. (1969). Cell lineage of the imaginal discs in Drosophila melanogaster. J. exp. Zool. 170, 61-76.

Hotta, Y. \& Benzer, S. (1972). Mapping of behaviour in Drosophila mosaics. Nature, Lond. 240, 527-535.

Hotta, Y. \& Benzer, S. (1973). Mapping of behaviour in Drosophila mosaics. In Genetic Mechanisms of development (ed. F. H. Ruddle), pp. 129-167. New York: Academic Press.

JANNING, W. (1975). Entwicklungsgenetische Untersuchungen an Gynandern von Drosophila melanogaster. III. Einige Beogachtungen an larvalen Gynandern. Verh. dt. zool. Ges. 1974, pp. 134-138.

JANNING, W. (1976). Entwicklungsgenetische Untersuchungen an Gynandern von Drosophila melanogaster. IV. Vergleich der morphogenetischen Anlagepläne larvaler und imaginaler Strukturen. Wilhem Roux Arch. EntwMech. Org. 179, 349-373.

Kankel, D. R. \& HaLl, J. C. (1976). Fate mapping of nervous system and other internal tissues in genetic mosaics of Drosophila melanogaster. Devl Biol. 48, 1-24.

Kaplan, W. D. \& Trout, W. E. III. (1969). The behaviour of four neurological mutants of Drosophila. Genetics, Princeton 61, 399-409.

Lewis, E. B. \& BACHER, F. (1968). Method of feeding ethylmethanesulphonate (EMS) to Drosophila males. Drosoph. Inf. Serv. 43, 193-194.

Lindsley, D. L. \& Grell, E. H. (1968). Genetic variations of Drosophila melanogaster. Carnegie Inst. Wash. Publ. 627.

Merriam, J. R. (1969). FM7: a 'new' first chromosome balancer. Drosoph. Inf. Serv. 44, 101. Miller, A. (1965). In Biology of Drosophila (ed. M. Demerec). New York: Hafner.

MintZ, B. \& BAKER, W. W. (1967). Normal mammalian muscle differentiation and gene control of isocitrate dehydrogenase synthesis. Proc. natn. Acad. Sci. U.S.A. 58, 592-598.

Nissani, M. (1975). Cell lineage analysis of kynurenine producing organs in Drosophila melangoaster. Genet. Res., Camb. 26, 63-72. 
Poulson, D. F. (1950). In Biology of Drosophila (ed. M. Demerec). New York: Hafner.

Pringle, J. W. S. (1957). Insect flight. Cambridge University Press.

Roth, R. L., Gomez, D., Voneida, T. J. \& Edwards, J. S. (1971). A modification of the Nauta technicile for freshwater teleosts and insects. In Contemporary Research Methods in Neuroanatomy (ed. W. J. H. Nauta \& S. O. E. Ebbesson). New York: Springer-Verlag.

SHAFIQ, S. A. (1963). Electron microscopic studies on the indirect flight muscles of Drosophila melanogaster. I. Structure of the myofibrils. J. Cell Biol. 17, 351-362.

SNOdgrass, R. E. (1935). Principles of Insect Morphology. New York: McGraw-Hill.

Sonnenblick, B. P. (1950). In Biology of Drosophila (ed. M. Demerec. New York: Hafner.

Stern, C. (1940). The prospective significance of imaginal discs in Drosophila. J. Morph.67, $107-128$.

Sturtevant, A. H. (1929). The claret mutant type of Drosophila simulans: a study of chromosomal elimination and of cell-lineage. Z. wiss. Zool. 135, 323-356.

TIEGS, O. W. (1955). The flight muscles of insects - their anatomy and histology; with some observations on the structure of striated muscle in general. Phil. Trans. R. Soc. B. 238, 221-348.

WhitTEN, J. M. (1962). Breakdown and formation of connective tissue in the pupal stage of an insect. Q. Jl Microsc. Sci. 103, 359-367.

Whitten, J. M. (1964). Haemocytes and the metamorphosing tissues in Sarcophaga bullata, Drosophila melanogaster, and other cyclorrhaphous Diptera. J. Insect Physiol. 10, 447-469.

(Received 22 September, 1976, revised 20 December 1976) 
